The Impact of Large-Scale Surveys on Pulsating Star Research ASP Conference Series, Vol. 203, 2000

L. Szabados \& D. W. Kurtz, eds.

\title{
Variable Star Research by the PLANET Collaboration
}

\author{
Michael D. Albrow ${ }^{1}$, K. R. Pollard ${ }^{1}$, J.-P. Beaulieu ${ }^{2}$, J. A. R. Caldwell ${ }^{3}$, \\ J. Menzies ${ }^{3}$, P. Vermaak ${ }^{3}$, D. L. DePoy ${ }^{4}$, B. S. Gaudi ${ }^{4}$, A. Gould ${ }^{4}$, \\ R. W. Pogge , M. Dominik ${ }^{5}$, R. M. Naber ${ }^{5}$, P. D. Sackett ${ }^{5}$, \\ J. Greenhill ${ }^{6}$, K. Hill ${ }^{6}$, S. Kane ${ }^{6}$, R. Watson ${ }^{6}$, R. Martin ${ }^{7}$, \\ A. Williams ${ }^{7}$, K. C. Sahu ${ }^{8}$ \\ (The PLANET collaboration ${ }^{9}$ )
}

\begin{abstract}
We review the current status and future prospects of the PLANET collaboration, an international team of astronomers performing high-precision photometric monitoring of microlensing events. Our photometric precision and sampling is characterised and the suitability of the database for variable star studies is discussed. Preliminary results on $\mathrm{K}$-giant stability are presented.
\end{abstract}

\section{Overview of PLANET}

PLANET (Probing Lensing Anomalies NETwork) was conceived in early 1995 to monitor intensively transitory microlensing events discovered by the microlensing survey groups. Since that time we have undertaken CCD photometry of these events using a network of telescopes, which currently include the 1-m telescope at the South African Astronomical Observatory, the Yale 1-m telescope at CTIO in Chile, the 1-m telescope in Hobart, Tasmania and the 0.6-m telescope at the Perth Observatory in Western Australia. Weather permitting, we can obtain nearly 24-hr continuous coverage of fields towards the Galactic Bulge during the Southern Hemisphere winter.

Recent results from PLANET have included prediction and observation of a second caustic crossing in MACHO 98-SMC-1 from which we inferred that the lens is most likely located in the SMC and not in the Galactic halo (Albrow et al. 1999a; Afonso et al. 1999); the first ever measurement of limb-darkening by microlensing (Albrow et al. 1999b); and the first limits on planetary companions to a Galactic bulge star (Gaudi et al. 1998; Albrow et al. 1999c).

\footnotetext{
${ }^{1}$ Department of Physics and Astronomy, University of Canterbury, Private Bag 4800, Christchurch, New Zealand

${ }^{2}$ Institut d'Astrophysique de Paris, INSU CNRS, 98 bis Boulevard Arago, F-75014, Paris, France

${ }^{3}$ South African Astronomical Observatory, P.O. Box 9, Observatory 7935, South Africa

${ }^{4}$ Ohio State University, Department of Astronomy, Columbus, OH 43210, USA

${ }^{5}$ Kapteyn Astronomical Institute, Postbus 800, 9700 AV Groningen, The Netherlands

${ }^{6}$ University of Tasmania, Physics Dept., G.P.O. 252C, Hobart, Tasmania 7001, Australia

${ }^{7}$ Perth Observatory, Walnut Road, Bickley, Perth 6076, Australia

${ }^{8}$ Space Telescope Science Institute, 3700 San Martin Drive, Baltimore, MD 21218, USA

${ }^{9} \mathrm{http}: / /$ www astro.rug. $\mathrm{nl} / \sim \mathrm{planet}$
} 


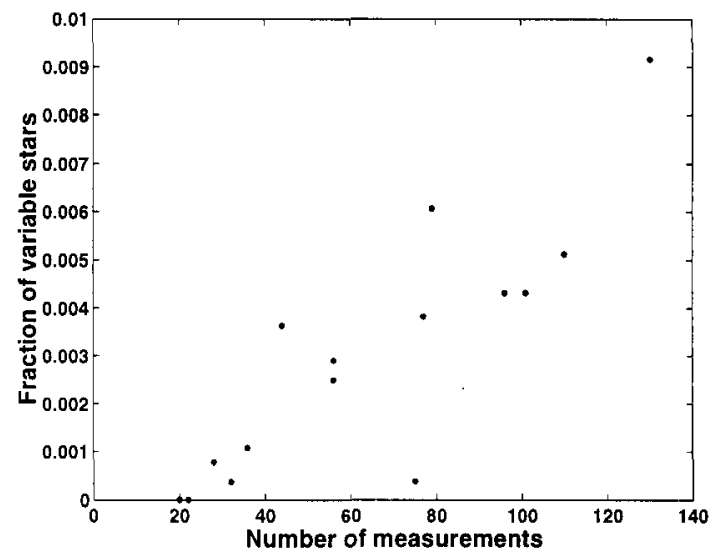

Figure 1. Detectability of variable stars in different fields from Yale 1-m $I$ band observations in 1998.

\section{Characterisation of PLANET Photometry}

PLANET is not a microlensing survey team. We react to microlensing alerts issued by the major surveys (OGLE, MACHO and EROS). Consequently, the fields we observe and our temporal sampling is governed by the nature of the microlensing events, not by any structured programme to observe variable stars. Currently most of our observation are in $I_{\mathrm{C}}$ with additional $V_{\mathrm{J}}$ observations. The majority of our target fields are towards the Galactic Bulge. In principle, our photometry is performed in near-real-time at the telescope using DoPhot (Schechter, Mateo, \& Saha 1993) in fixed position mode.

PLANET observations for 1998 are summarised in Table 1. The SAAO and CTIO data have a photometric error reported by DoPhot of a few percent at $I=$ 18 and $V=20$. Our fields tend to be crowded - this is the primary factor limiting our photometric accuracy. Currently, the data from different sites are analysed separately; cross-referencing and calibration for individual fields between sites is in progress.

Table 1. PLANET observing statistics for 1998

\begin{tabular}{lccc}
\hline & $I$ frames & $V$ frames & Fields \\
\hline SAAO & 2988 & 686 & 41 \\
CTIO & 1097 & 747 & 17 \\
Tasmania & 631 & 123 & 11 \\
Perth & 299 & 55 & 16 \\
\hline \multicolumn{4}{r}{} \\
\hline
\end{tabular}



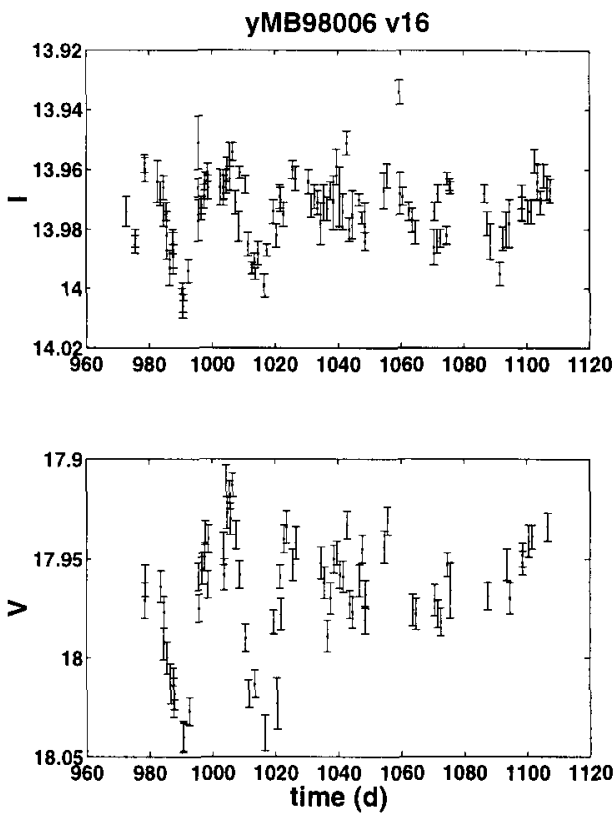

Figure 2. An example of a semiregular star identified as a variable in both the $I$ and $V$ bands. The $I$ band full amplitude is only $\sim 40 \mathrm{mmag}$. The $V$ band full amplitude is $\sim 0.1 \mathrm{mag}$ but the magnitude is faint due to heavy interstellar and/or circumstellar reddening.

\section{Periodic Variable Stars}

All fields are searched for variable stars using the AoV method (SchwarzenbergCzerny 1989). As shown in Fig. 1, the majority of fields are undersampled. An example of our sensitivity to variable stars is shown in Fig. 2 where a semiregular variable is detected in both $I$ and $V$ with full amplitudes of 0.04 mag at $I=14$ and $0.1 \mathrm{mag}$ at $V=18$.

The period-amplitude distribution of variables found in the Yale 1-m data from 1998 is shown in Fig. 3. At the short period end of the distribution the majority are RR Lyrae stars, while at longer periods (which are often not well defined by our data) we find many long period and semiregular variables, usually heavily reddened.

Fig. 4 shows the location of the variables detected in the colour-magnitude diagram for one field. Long period and semiregular variables predominate, but there are a number of variables around the red clump, some of which may be rotating ellipsoidal or spotted stars. 


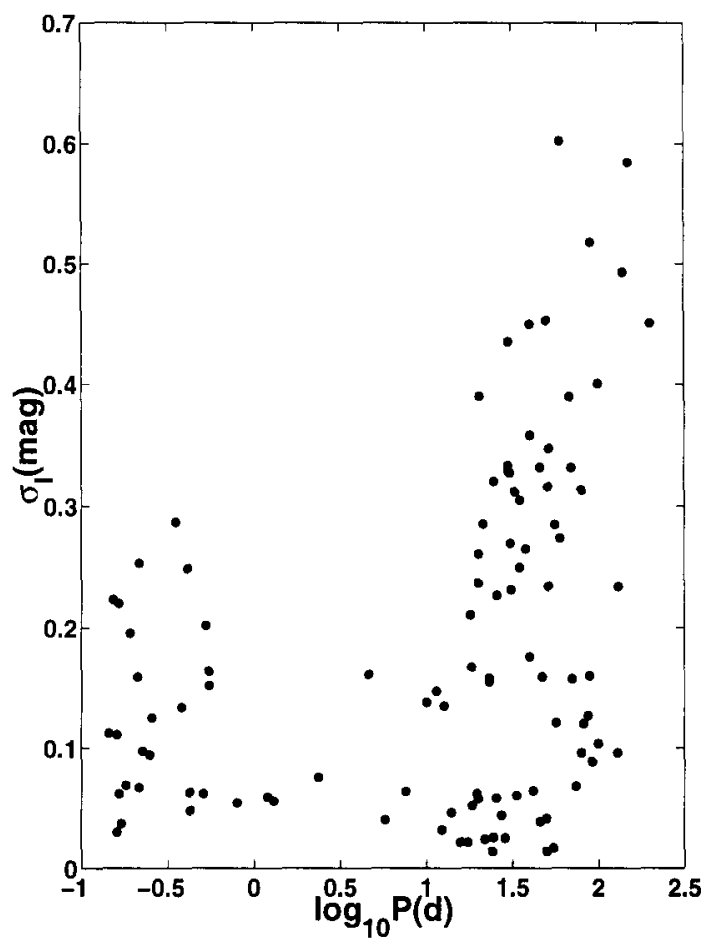

Figure 3. Period-amplitude distribution of the variable stars found in the Yale 1-m fields in 1998.

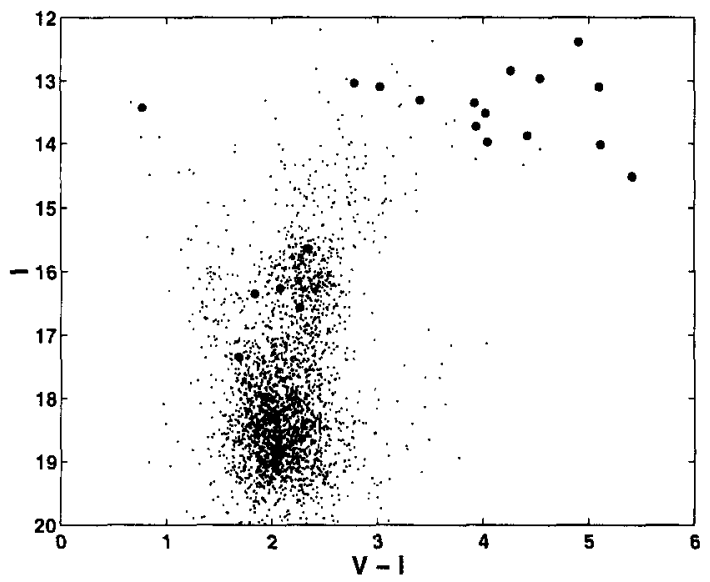

Figure 4. Colour-magnitude diagram of the best-sampled Yale 1 - $\mathrm{m}$ field in 1998 showing the locations of the periodic variable stars detected. 


\section{Stability of K Giants}

We have recently begun an investigation into the photometric stability of red giant stars in the Bulge. Recent investigations have suggested that a fraction of $\mathrm{K}$ giant stars are pulsationally unstable at about the $10 \mathrm{mmag}$ level in $V$ (Edmonds \& Gilliland 1996; Henry et al. 1999). Aside from pulsational studies, this is important because microlensed sources are commonly red giants and any intrinsic photometric variability could be misidentified as small deviations induced by lens or source binarity. For example, a (somewhat controversial) apparent deviation of $\sim 2 \%$ in the event MACHO 98-BLG-035 has recently been suggested to be due to a low-mass planetary companion (Rhie et al. 1999).

From our best-sampled Yale 1-m field from 1998, we have selected those stars in and around the red clump that have at least 20 measurements in both the $I$ and $V$ bands with seeing better than $2.5^{\prime \prime}$ and formal DoPhot uncertainty less than $15 \mathrm{mmag}$. Empirical seeing corrections were made by fitting low-order polynomials to the photometry as a function of seeing for each of the 205 stars in the sample. The standard deviations of the photometric magnitudes are plotted in Fig. 5. No periodicity was found for these stars. Clump giants thus appear to be photometrically stable to the $4 \%$ level or less, but the correlation between the $I$ and $V$ photometry (despite its not being simultaneous) suggests that a subset of these giants may be variable at a few percent level.

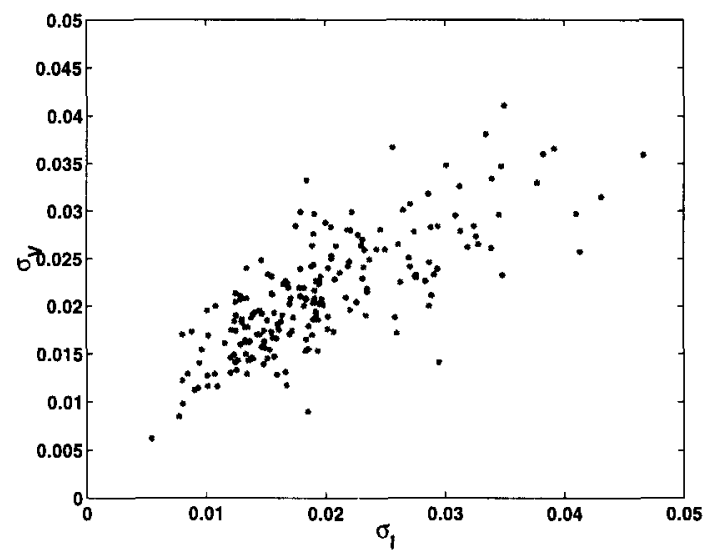

Figure 5. Photometric stability in $I$ and $V$ bands of red clump stars from the best-sampled Yale 1-m field from 1998.

\section{Future Plans}

Two major changes that will affect our future research are in the areas of photometric reduction and filters. Currently we are experimenting with reducing our data using the image subtraction package ISIS2 (Alard 1999). There are indications that this will reduce both random and systematic errors in our photometry. We are also beginning to use new cameras at CTIO and SAAO which have a 
dichroic beam-splitter and can image simultaneously in the optical and infrared. From 2000, most of our photometry from these sites will include simultaneous near-IR photometry, most likely in the $H$ band.

\section{References}

Afonso, C., Alard, C.. Albert, J. N., et al. 1999, ApJ, submitted, [astro$\mathrm{ph} / 9907247\}$

Alard, C. 1999, A\&A, submitted, [astro-ph/9903111]

Albrow, M. D., Beaulieu, J.-P., Caldwell, J. A. R., et al. 1999a, ApJ, 512, 672

Albrow, M. D., Beaulieu, J.-P., Caldwell, J. A. R., et al. 1999b, ApJ, 522, 1011

Albrow, M. D., Beaulieu, J.-P., Caldwell, J. A. R., et al. 1999c, ApJ, submitted, [astro-ph/9909325]

Edmonds, P. D. \& Gilliland, R. L. 1996, ApJ, 464, L157

Gaudi, B. S., Albrow, M. D., Beaulieu, J.-P., et al. 1998, AAS, 193, 108.07

Henry, G. W.. Fekel F. C.. Henry, S. M., \& Hall, D. S. 1999, preprint

Rhie, S. H., Bennett, D. P., Becker, A. C., et al. 1999, ApJ, submitted, [astroph/9905151]

Schechter, P. L., Mateo M., \& Saha, A. 1993, PASP, 105, 1342

Schwarzenberg-Czerny, A. 1989, MNRAS, 241, 153

\section{Discussion}

Piotr Popouski: What is PLANET going to do when MACHO goes off-line and they miss most of their alerts?

Michael Albrow: Currently about half the events we follow are alerted by MACHO, and the other half by OGLE. Next year there will be no MACHO alerts, but OGLE expect that their alert rate will increase by a factor of four when their new camera is installed. 